\title{
ROTEIRIZAÇÃO DE VEÍCULOS PARA TRANSPORTE DE FUNCIONÁRIOS - ESTUDO DE CASO EM PERNAMBUCO
}

\author{
Caio Bezerra Souto Maior \\ caio.maior@ufpe.br; caiomaior@hotmail.com \\ Isis Didier Lins \\ isis.lins@ceerma.org \\ Márcio das Chagas Moura \\ marcio@ceerma.org \\ Débora Mendonça de Santana \\ deboramsantana4@gmail.com
}

Centro de Estudos e Ensaios em Risco e Modelagem Ambiental - CEERMA

Departamento de Engenharia de Produção, Universidade Federal de Pernambuco - UFPE

Av. Prof. Moraes Rego, 1235 - Cidade Universitária, Recife - PE - Brasil - 50670-901

\section{RESUMO}

O crescimento de empresas e indústrias mais distantes dos centros urbanos trouxe uma preocupação por parte dos empresários em como viabilizar o transporte dos seus funcionários até as empresas. Este trabalho procura, a partir da aplicação de modelos matemáticos, criar rotas otimizadas para o deslocamento dos funcionários no trajeto residência-trabalho e vice-versa. Para isso, o trabalho está dividido em três etapas. Primeiramente, uma análise de clusters é realizada através do software R-Studio para facilitar a definição dos pontos de parada onde cada funcionário será alocado. Depois de determinados os clusters, os pontos de parada são definidos de forma visual, e por último, um modelo de roteirização é resolvido de forma tanto de forma exata por meio do IBM ILOG CPLEX como de forma aproximada utilizando o método da "varredura" e a heurística do vizinho mais próximo. $\mathrm{O}$ exemplo de aplicação utilizou 50 funcionários, os quais foram divididos em 10 clusters com 5 membros em cada. Um total de 14 pontos de parada foram definidos. Foram simuladas quatro situações com diferentes capacidades de veículos. A distância total percorrida, os custos e o tempo foram analisados em cada simulação e comparados entre si para então definir-se a quantidade e a capacidade dos veículos utilizados e as rotas que cada veículo irá percorrer.

Palavra-chave: Análise de clusters; definição de pontos de parada; roteirização; transporte de funcionários;

ABSTRACT 
The growth of companies and industries farther from urban centers has raised a concern on the part of entrepreneurs on how to make it possible for their employees to be transported to companies. This paper aims, through the application of mathematical models, to create optimized routes for the displacement of employees in the home-work course and vice versa. For this, the work is divided into three steps. First, cluster analysis is performed using R-Studio software to make it easier to define breakpoints where each employee will be allocated. Once the clusters are determined, the breakpoints are defined visually, and lastly, a routing model is solved both accurately through IBM ILOG CPLEX and roughly using the "sweep" method and the nearest neighbour heuristic. The application example used 50 employees, which were divided into 10 clusters with 5 members each. A total of 14 stopping points has been set. Four situations with different vehicle capacities were simulated. The total distance traveled, costs and time spent were analyzed in each simulation and compared to each other to define the quantity and capacity of the vehicles used and the routes each vehicle will travel.

Keywords: Cluster analysis; definition of stopping points; routing; transportation of employees. 


\section{INTRODUÇÃO}

Nos últimos anos, a intensa industrialização brasileira e, especificamente, a pernambucana, trouxe grandes fábricas para entornos de centros urbanos interioranos muitas vezes longe da região metropolitana do Recife. Este afastamento da capital e dos espaços urbanizados dá por diversos motivos como a dificuldade de encontrar espaços livres para a construção das fábricas dentro das metrópoles e a implementação de leis ambientais e sanitárias que exigem um distanciamento mínimo de locais muito povoados.

Especificamente, o meio de locomoção dos funcionários deve ser estudado pelas empresas, já que a Lei $\mathrm{n}^{\circ} 7.418$, art. $1^{\circ}$ [1] exige que o empregador disponibilize um valetransporte ao funcionário para utilização com despesas de deslocamento residência-trabalho e vice-versa. Porém, esse vale-transporte nem sempre é eficaz por não haver frequentes rotas de transportes coletivos públicos passando por regiões afastadas dos centros urbanos. Assim, cabe ao empregador viabilizar alguma outra forma de transporte para os funcionários.

Nesse contexto, o incentivo ao uso do carro particular pode ser uma das soluções. Entretanto, o alto custo por parte das empresas ao pagar o combustível dos trabalhadores pode tornar essa solução ineficaz. Além disso, essas indústrias contratam centenas de funcionários de diferentes níveis sociais, o que faz com o que o acesso ao carro particular possa não ser possível para todos. Uma solução utilizada por várias empresas é a contratação de ônibus que buscam seus funcionários em suas casas, ou o mais próximo delas possível, e as deixa de volta ao final do expediente. Por outro lado, essa alternativa pode perder sua eficácia se não houver um estudo adequado, garantindo a eficiência no serviço de transporte dos funcionários. Por exemplo, se a empresa contratar mais ônibus do que o necessário, haverá um aumento de custo assim como se a definição de rotas que não possuírem os menores percursos, gerando um maior tempo de deslocamento e um maior custo com combustível. Assim, é preciso definir pontos estratégicos de parada dos ônibus buscando juntar o maior número de funcionários possível em cada ponto, diminuindo o tempo total de rotas.

Esse trabalho foi dividido em três fases. A primeira etapa foi definir os pontos de paradas dos funcionários utilizando uma análise de clusters. A análise de cluster, segundo [2], é uma técnica analítica de criar a partir de um grupo maior de indivíduos ou objetos, subgrupos menores que sejam similares entre si. Após a divisão dos clusters, à segunda etapa definiu os pontos de parada de forma visual através da ferramenta Google Maps. A última etapa consiste em determinar as rotas de cada veículo, e fazendo parte do conhecido problema de roteirização, muito comum na de logística.

\section{REFERENCIAL TEÓRICO}

\subsection{OTIMIZAÇÃo LOGÍSTICA}

Segundo [3], o rápido desenvolvimento tecnológico, a globalização e a maior exigência dos clientes estão tornando o mercado cada dia mais competitivo. Esse aumento da competitividade exigiu uma busca por algum diferencial por parte das empresas para manter e atrair novos clientes, muitas vezes sendo alcançada com investimentos na logística para conseguir entregar o produto certo, na hora certa e de maneira correta.

A logística pode ser entendida como uma parte do processo do gerenciamento da cadeia de suprimentos que visa a satisfação do cliente com o menor custo através da melhoria do nível de serviço. Nesse contexto, a gestão da cadeia de suprimentos, segundo [4], é "a gestão de interconexão das empresas que se relacionam entre si por meio de ligações a montante e a jusante entre os diferentes processos, que produzem valor na forma de produtos e serviços para o consumidor final".

Assim, decisões sobre a localização das instalações, os estoques e o transporte de 
bens e materiais são preocupações da logística e da gestão da cadeia de suprimentos, pois impactam diretamente o nível de serviço ao cliente. É importante ressaltar que o transporte é a atividade de maior custo e decisões estratégicas (ex. escolha do tipo de modal utilizado, melhores rotas percorridas) têm importante significância dentro do planejamento logístico e constituem problemas muito frequentes de tomada de decisão [5].

Os problemas de roteirização podem ser de diferentes tipos como: 1) ponto de origem diferente do ponto de destino; 2) mais de um ponto de destino e de origem; e 3) pontos de destino e origem iguais. Assim, existem métodos específicos para cada um desses problemas. Um método utilizado para resolver o primeiro grupo de problemas, por exemplo, é o do caminho mais curto. Em problemas com múltiplos pontos de origem e destino, é comum o uso do método do transporte, enquanto que a última classe de problemas o problema do caixeiro viajante pode ser estruturado. O problema de roteirização é uma generalização do caixeiro viajante, agora considerando mais de um "caixeiro", cada um com uma capacidade máxima e demanda específica em cada nó.

O problema de roteirização pertence à classe NP de problemas, ou seja, à medida em que o tamanho do problema aumenta (número de cidades, clientes ou nós), o tempo de execução para resolvê-lo aumenta de maneira polinomial não determinística. Com isso, se a instância do problema for grande, não é possível resolvê-lo de forma exata em tempo hábil e é necessário utilizar métodos heurísticos ou meta-heurísticos, como algoritmo genético e colônia de formigas. Nesse contexto, a Pesquisa Operacional (PO) é utilizada como ferramenta para resolução desse tipo de problemas, auxiliando o processo de tomada de decisões em situações que requerem alocações eficientes de recursos escassos. Assim, primeiramente, fazse necessária a modelagem do problema que se quer resolver por meio da definição das variáveis, parâmetros e relações matemáticas. Após a criação do modelo é necessário aplicar ferramentas matemáticas para resolvê-lo. Depois de obtidos os resultados, uma avaliação é necessária para interpretá-los e para verificar se são adequados ao contexto de aplicação [6]. Abaixo estão descritos conceitos e definições sobre caixeiro viajante e roteirização de veículos necessários para a construção do modelo apresentado neste trabalho.

\subsubsection{Problema do caixeiro-viajante}

O problema envolve um caixeiro, que sairá de uma cidade de origem e visitará um conjunto de cidades (nós) em apenas uma viagem, retornando à cidade base. Busca-se minimizar um ou mais objetivos dependendo do tipo do problema, mas o mais comum quando se considera como único objetivo a ser minimizado a distância total percorrida. Este problema possui várias formulações diferentes, uma delas é a de [7] apresentada abaixo:

$\min$

$$
\sum_{i=1}^{n} \sum_{j=1}^{n} C_{i j} X_{i j}
$$

S.a

$$
\begin{gathered}
\sum_{i=1}^{n} X_{i j}=1, i=1, \ldots, n \\
\sum_{j=1}^{n} X_{i j}=1, j=1, \ldots, n \\
U_{i}-U_{j}+(n-1) * X_{i j} \leq n-2, \quad i, i=2, \ldots n, \quad i \neq j \\
U_{1}=1
\end{gathered}
$$




$$
2 \leq U_{i} \leq n, \quad i=2, \ldots, n
$$

A função objetivo busca minimizar a distância total percorrida por todos os veículos juntos. Neste caso, $C_{i j}$ representa a distância entre os nós $i$ e $j$, e o $X_{i j}$ é uma variável binária que assume o valor 1 quando o caixeiro percorre o percurso $i j$ e 0 quando ele não percorre.As restrições asseguram que:

- O caixeiro só chega uma vez a cada cidade (Restrição 2);

- O caixeiro só deixa a cidade uma única vez (Restrição 3).

As funções 4, 5 e 6 garantem a eliminação de sub-rotas. $O U_{i}$ indica a posição na qual a cidade $i$ será visitada. $\mathrm{O} U_{1}$ será igual a 1 para indicar que o caixeiro inicia seu trajeto na cidade 1, sem perda de generalidade. Um dos métodos mais simples para resolver o problema do caixeiro viajante é o do vizinho mais próximo. Sua sistemática é: um ponto é escolhido como inicial, e toma-se como segundo ponto aquele que for mais próximo do primeiro. Faz-se o mesmo procedimento com o segundo ponto até todos os pontos estarem incluídos na rota. [8].

\subsubsection{Roteirização de veículos}

O problema de roteirização de veículos se assemelha ao problema do caixeiro viajante, porém busca definir rotas partindo de um ou mais pontos de depósitos até vários pontos de destino (clientes) de forma a minimizar o custo. O problema de roteirização de veículos utilizado aqui apresentado é definido por um grafo $G=(N, E)$, onde $N=C \cup$ $\{0\}, C=\{1, \ldots, n\}$ é o conjunto de nós representando os pontos de parada, e 0 representa o ponto de origem (sede da empresa). O conjunto $E=\{(i, j): i, j \in N, i \neq j\}$ é formado pelos arcos que conectam os nós. Todas as rotas começam em $0 . D_{i j}$ representa a distância necessária para ir de $i$ até $j$. Há um conjunto $Z$ de veículos idênticos, e cada veículo $z \in Z$ possui uma capacidade Q. $Y_{k}$ é uma variável binária que assumirá o valor 1 se o veículo $z$ for utilizado e 0 caso contrário. $X_{i j z}$ é uma variável binária que assumirá o valor 1 se o veículo $z$ percorrer o arco $(i, j)$, e será 0 , caso isso não ocorra. As restrições do problema são:

- Cada rota inicia no depósito;

- Cada cliente só pode estar incluído em uma rota;

- A demanda total de uma rota não pode exceder a capacidade $Q$ do veículo;

A função objetivo deste problema, que representa a minimização da distância total percorrida é:

$\min$

$$
\begin{gathered}
\sum_{z \in Z} \sum_{(i, j) \in E} D_{i j} X_{i j z} \\
\sum_{z \in Z} \sum_{j \in N} x_{i j k}=1, \forall \in C \\
\sum_{i \in C} d_{i} \sum_{j \in N} X_{i j z}<Q * Y_{k}, \forall z \in Z \\
\sum_{j \in N} X_{0 j z}=Y_{k}, \forall z \in Z \\
\sum_{i \in N} X_{i h z}-\sum_{j \in N} X_{h j z}=0 \forall h \in C, \forall z \in Z
\end{gathered}
$$




$$
\sum_{i \in C} X_{i 0 z}=Y_{k}, \forall z \in Z
$$

Cada função restrição assegura algo:

- Cada ponto de parada $i$ é visitado uma única vez e por um único veículo $z$ (Restrição 8);

- A demanda total de cada rota não pode ultrapassar a capacidade $Q$ do veículo (Restrição 9);

- Cada veículo $z$ só pode deixar o nó de origem (nó 0) se o mesmo for utilizado (Restrição 10);

- Cada veículo $z$ só pode deixar o nó $h$ se, e somente se, chegar a este nó (Restrição 11);

- Cada veículo $z$ só pode retornar ao nó de origem (nó 0) se o mesmo for utilizado (Restrição 12).

Para eliminar as sub-rotas foi utilizado a formulação de [7] com algumas alterações. As funções utilizadas para essa eliminação foram:

$$
\begin{gathered}
u_{0 k}=0, \forall k \in K \\
u_{i \in C, j \in N, i \neq j} X_{i j k}, \forall k \in K \\
u_{i k} \geq \sum_{j \in N, i \neq j} X_{i j k}, \forall k \in K \\
u_{i k}-u_{j k}+n * X_{i j k}+(n-2) * X_{i j k} \leq n-1, \forall i, j \in C, \forall k \in K, i \neq j \\
\sum_{p \in k, p \neq k} u_{i p} \leq n *\left(1-\sum_{j \in N, i \neq j} X_{i j k}\right), \forall k \in K, \forall i \in C
\end{gathered}
$$

- Todos os veículos $z$ estão no depósito no início (Restrição 13);

- O limite superior da ordem de visitação é o número de clientes visitados pelo veículo $z$ (Restrição 14); se $z$ não visita cliente algum, limite superior é 0 ;

- O Limite inferior da ordem de visitação será 1 , se o veículo $z$ visita o cliente $i$ (Restrição 15); se o veículo z não visita cliente algum, limite inferior é 0 ;

- Eliminação de sub-rotas para cada veículo $z$ (Restrição 16);

- Se o cliente $i$ é visitado pelo veículo $z$, a soma de $u_{i p}$ para $p \neq z$ deve ser 0 (Restrição 17);

A restrição 9 sofre uma alteração quando o problema tem uma frota com veículos de diferentes capacidades. A constante $Q$ precisa ser transformada em um vetor $Q_{z}$, que representará a capacidade de cada veículo $z$. O problema de roteirização de veículos, como visto acima, tem como função minimizar rotas no transporte de funcionários. Dessa forma, faz-se o planejamento das frotas de ônibus que buscam os funcionários em seus pontos de paradas e os levam até a empresa respeitando a capacidade máxima de cada veículo. Antes de resolver o problema da roteirização, é preciso determinar os pontos de parada a que cada funcionário será alocado. Essa etapa envolve a análise de clusters comentada na próxima seção.

Um método simples e rápido utilizado para resolver o problema de roteirização de 
veículos é o método da "varredura" [5], tendo o seu passo-a-passo descrito na Figura 2.1:

1. Coloque todas as paradas, inclusive o depósito, em um mapa;

2. Trace uma linha em qualquer direção a partir do depósito. Gire essa linha no sentido horário até ela interceptar uma parada. Os pontos que são interceptados pela linha vão sendo adicionados à rota até atingir a capacidade máxima do veículo. Comece uma nova rota com o próximo ponto, e continue a varredura até todos os pontos estarem em uma rota.

3. Dentro de cada rota aplique algum método que resolva o problema do "caixeiro viajante" para reduzir as distâncias percorridas.

Figura 2.1 - Método da "varredura"
Fonte: [5] Ballou (2006)

\subsection{ANÁLISE DE CLUSTERS}

Análise de clusters é a divisão de um conjunto de elementos em grupos menores, sendo os estes elementos dentro de seu grupo e diferentes dos outros grupos [9]. Existem diferentes métodos de análise de clusters, sendo necessário analisar o tipo de cluster para se definir qual método é o mais adequado ao contexto de aplicação considerado.

O algoritmo não-hierárquico K-means, segundo [10], é um dos mais conhecidos, possuindo diversas variantes. Na versão básica, o número K de clusters é definido inicialmente e um conjunto $\mathrm{K}$ de centros são escolhidos aleatoriamente. Cada ponto restante é atribuído ao cluster no qual o centro K está mais perto em distância Euclidiana, e o novo centro de cada cluster é calculado. Tal processo o é repetido até que nenhum elemento altere de grupo.

Os métodos hierárquicos podem ser divididos em duas formas: divisiva e aglomerativa. As divisivas começam com apenas um grande grupo composto por todos os elementos e vão dividindo-o em grupos menores até cada cluster conter apenar um ponto. Já a versão aglomerativa, mais utilizada que a anterior, começa com grupos isolados com apenas um elemento que vão se juntando até formar um único grupo com todos os pontos. $\mathrm{O}$ algoritmo na forma aglomerativa segue o passo-a-passo descrito na Figura 2.2.

1. Faça um cluster para cada elemento;

2. Encontre os pares de clusters mais similares de acordo com uma medida de distância determinada;

3. Junte os clusters que compõem cada par definido no passo 2 em um cluster maior e recalcule a distância entre o novo cluster e os demais;

4. Repita os passos 2 e 3 até existir apenas um único cluster.

Figura 2.2 - Método hierárquico na versão aglomerativa

Fonte: [10]

Há três formas diferentes de calcular a distância entre dois clusters:

- Single link: A distância entre dois clusters é a distância entre os seus elementos mais próximos.

- Average link: A distância entre dois clusters é a distância entre os centroides dos dois clusters.

- Complete link: A distância entre dois clusters é a distância entre os seus elementos mais distantes.

Neste trabalho, a análise de clusters será útil para dividir as residências dos funcionários em grupos e facilitar a definição dos pontos de paradas.

\subsection{REVISÃo DA LITERATURA}

[11] destaca o problema de roteirização de ônibus escolar como uma variante do problema de roteirização de veículos em que três decisões simultâneas têm de ser feitas: (1) 
determinar o conjunto de paradas para visitar, (2) determinar, para cada estudante até que ponto de parada ele deve andar, e (3) determinar as rotas que se encontram ao longo das paradas escolhidas, de modo que a distância percorrida total é minimizada. Para solucionar este problema, um procedimento meta-heurístico foi desenvolvido, utilizando a construção de um Greedy Randomised Adaptative Search Procedure (GRASP) seguido por um Variable Neighborhood Descent (VND). Experimentos realizados em 112 casos mostraram que o GRASP e o VND encontram soluções ótimas muito mais rápido que o procedimento exato e pode lidar com casos que são muito maiores.

Segundo [12], o GRASP é uma técnica iterativa que se inicia a partir da solução de uma função aleatória gulosa. Em seguida, aplica-se um procedimento de busca local à solução encontrada buscando alguma uma melhoria. Ele baseia-se no método das economias de Clarke \& Wright, muito utilizado na resolução de problemas de roteirização devido à simplicidade de implementação e rapidez nos cálculos [13]. Já o VND, de acordo com [14], parte de uma solução inicial e a compara com os vizinhos um a um. Quando uma solução melhor é encontrada, aquela solução inicial é substituída por essa melhor.

[15] criou um software para automatizar o processo de construção e atualização das rotas dos ônibus fretados que fazem o transporte dos funcionários no trajeto residênciaUNICAMP (Universidade Estadual de Campinas) e vice-versa. Quando um funcionário é adicionado ou removido ou se quer apenas alterar uma rota, o software criado utiliza uma meta-heurística que explora os conceitos de Large Neighborhood Search (LNS) e Variable Neighborhood Search (VNS) para resolver o problema do caixeiro comprador. Segundo [16], esse problema busca definir uma rota otimizada para aquisição de produtos. Dessa forma, o valor e quantidade do produto devem ser levados em consideração na escolha da rota já que existem mercados com preços, quantidades e localizações diferentes, com o comprador atendendo às demandas por cada produto com o objetivo de minimizar a soma do custo de compra e de deslocamento. No caso de querer alterar/criar mais de uma rota, o software resolve o problema dos $m$ caixeiros compradores, semelhante ao problema do Caixeiro Comprador, porém considerando mais de um caixeiro.

Em seu trabalho, [17] compara a heurística de Clark \& Wright e o algoritmo genético para a solução do problema de roteamento de veículos com restrição de capacidade. Os critérios utilizados para esta comparação foram a qualidade nas respostas encontradas e o tempo de execução, e o algoritmo de Clark \& Wright teve uma melhor performance do que o algoritmo genético.

[18] aplicaram, em seu trabalho, técnicas heurísticas para resolver o problema do transporte escolar em 32 munícipios do Estado do Paraná. Eles dividiram o trabalho em três etapas: 1) definiram os pontos de parada de acordo com as residências dos alunos, que foram designados a seu respectivo ponto de parada respeitando uma distância máxima permitida; 2) calcularam as distâncias reais entre os pontos de parada e as escolas; e 3) construíram as rotas através do algoritmo Adapted Location Based Heuristic. Houve uma economia para estes municípios que variou entre $3,1 \%$ e $33,55 \%$ na quilometragem total diária, e redução da quantidade de veículos utilizados. Além disso, em duas cidades houve uma diminuição considerável do número de pontos de parada.

Portanto, é possível notar que existe uma enorme variação nos diferentes tipos de problemas de roteirização com novos métodos criados com base nos já existentes. O maior problema encontrado em empresas não é a falta de opções para solucionar seus problemas de roteirização, mas sim a falta de investimento nesta área.

O presente trabalho se assemelha com alguns dos trabalhos citados acima pelo fato de todos eles estarem preocupados com o transporte de pessoas, entretanto, o presente trabalho resolverá este problema de forma exata, já que a instância considerada é relativamente pequena e a aplicação de heurísticas não é justificada. Além disso, será aplicado no contexto de 
transporte de funcionários de uma empresa fictícia, como será mostrado no próximo capítulo.

\section{EXEMPLO DE APLICAÇÃO}

\subsection{MÉTODO DE RESOLUÇÃO}

O problema deste trabalho foi dividido em três etapas, apresentados no fluxograma da Figura 3.1:

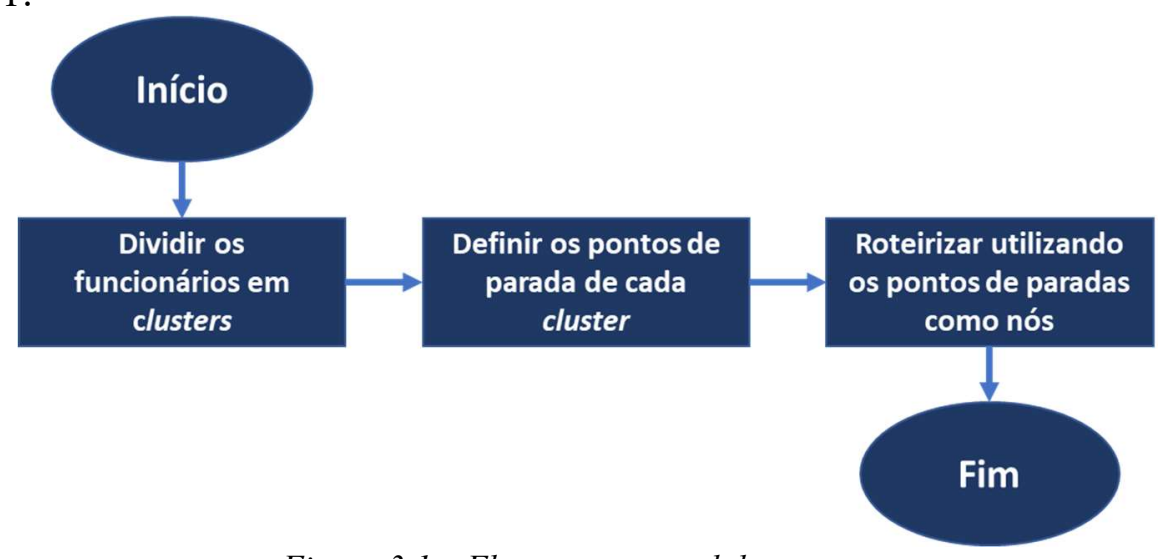

Figura 3.1 - Fluxograma geral das etapas

Fonte: O autor (2019)

A primeira etapa foi dividir os funcionários em clusters de acordo com a localização de suas respectivas residências. Para dar início a esta etapa, foi necessário criar uma matriz de distâncias entre todos os 51 pontos (50 residências dos funcionários e a empresa). Por haver uma grande distorção entre a distância real percorrida por um veículo e a distância Euclidiana, as distâncias reais foram adotadas neste trabalho. Elas foram obtidas por meio da ferramenta Google Maps.

A segunda parte foi definir os pontos de paradas e os funcionários alocados a cada ponto de acordo com os clusters formados na etapa anterior. Na última, foi realizada a roteirização dos veículos utilizando os pontos de parada definidos anteriormente. Como resposta final, obteve-se as rotas de cada veículo de modo a minimizar a distância total percorrida. A seguir cada uma das etapas é detalhada.

\subsection{FORMAÇÃO DOS CLUSTERS}

Para a criação dos pontos de parada, aplicou-se a análise de cluster por meio de uma variante do algoritmo aglomerativo que buscava facilitar o cálculo do ponto de gravidade. Apesar desse cálculo ter se mostrado ineficiente, como verificado na próxima seção, a análise de clusters continuou sendo útil já que a definição dos pontos de parada foi feita de forma visual através do Google Maps, e seria inviável visualizar os 50 pontos de residência dos funcionários ao mesmo tempo. $\mathrm{O}$ algoritmo aglomerativo não permite que a quantidade de elementos em um grupo ultrapasse o valor 5. A escolha deste valor se deu por dois motivos: primeiramente, para facilitar a visualização dos pontos no Google Maps e segundo porque permitiria formar 10 grupos com exatamente 5 pontos em cada. A Figura 3.2 mostra as etapas realizadas nesta análise.

O algoritmo inicia selecionando o menor valor na matriz de distância, e os pontos que estão na linha e coluna deste valor formam, neste momento, um único cluster. Calcula-se as novas distâncias entre este novo grupo e os outros pontos utilizando a distância single link, a menor distância de cada ponto para cada ponto do cluster. A nova menor distância é selecionada e, se um dos pontos desta nova distância já estiver em algum cluster que não tenha cinco pontos, o outro ponto é adicionado neste cluster. Se os dois pontos já estiverem alocados em diferentes clusters, e os clusters desses dois pontos somados não excederem a capacidade 
total para cada cluster, os dois clusters se juntam formando um novo. Se os dois pontos ainda não foram alocados em nenhum cluster, um novo cluster é criado com estes dois pontos. Este processo se repete até o momento em que todos os pontos estiverem alocados. Para a realização desta etapa foi utilizado o programa R-Studio.

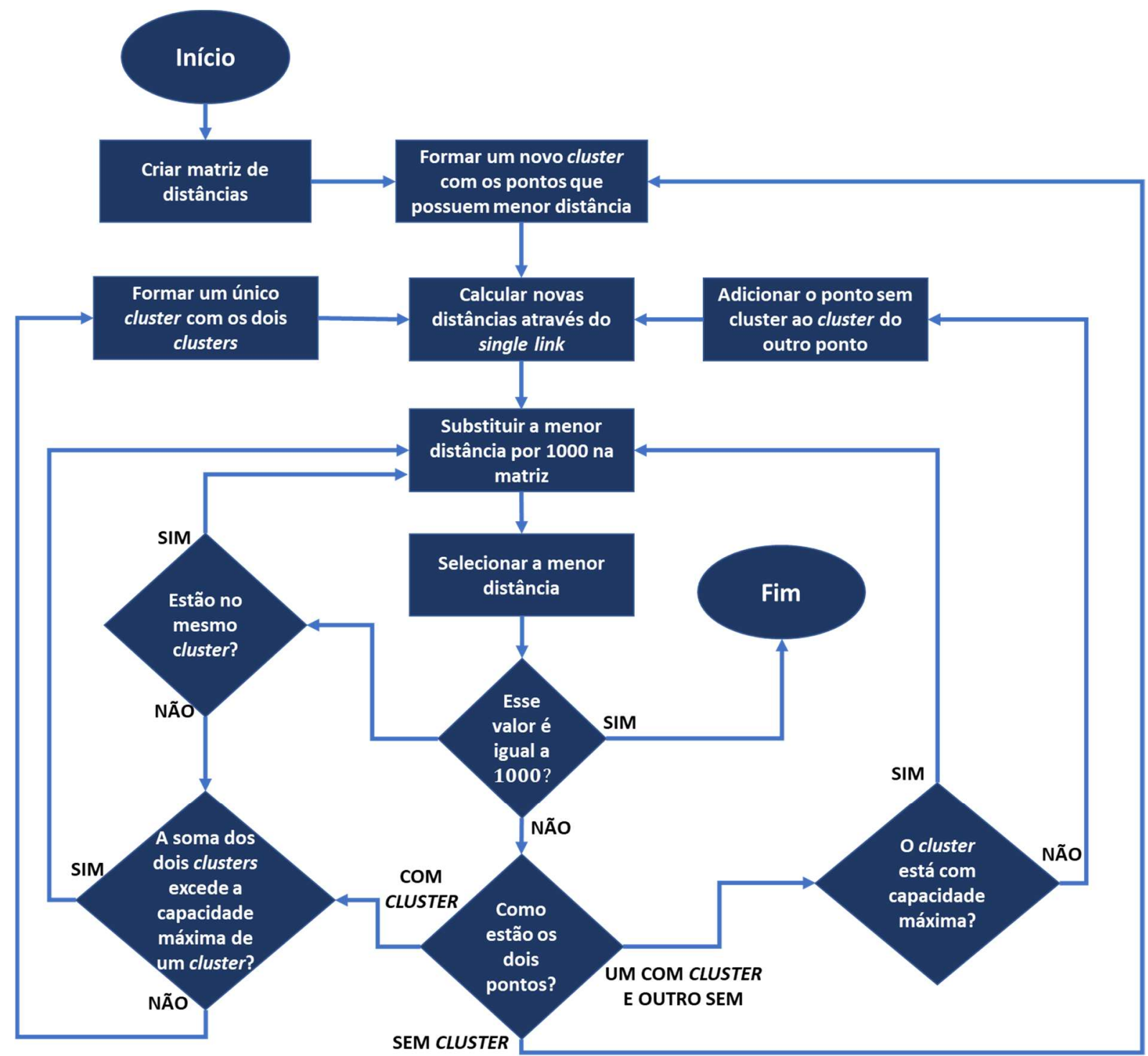

Figura 3.2 - Fluxograma da análise de clusters

Fonte: $O$ autor (2019)

\subsection{DEFINIÇÃO DOS PONTOS DE PARADA}

Depois de definidos os clusters, com no máximo 5 pontos, os endereços foram colocados no Google Maps para calcular o ponto de gravidade [5] associado a cada grupo e, assim, definir os pontos de parada.

Esta abordagem inicial não se mostrou eficiente pois, em muitos casos, os centros de gravidade se localizavam em uma rua menor, que impossibilitava ou dificultava a parada de um veículo grande como um ônibus, ou mesmo em cima de uma construção.

A solução encontrada foi descartar os pontos de gravidade e definir os pontos de parada em avenidas ou ruas maiores que se encontrassem entre os 5 funcionários do cluster. Como em alguns casos as moradias ainda eram distantes entre si, foi necessário obter mais de 
um ponto de parada por cluster. Ainda, houve situações em que determinados pontos ficariam penas com um funcionário. Neste caso, tais empregados foram alocados para outras paradas e receberiam auxílio transporte para chegar até lá com o transporte público.

Quando selecionados, cada ponto de parada precisava se localizar a, no máximo, 850 metros das residências, caso o funcionário fosse a pé, uma distância razoável para se caminhar, das residências dos funcionários que seriam alocados a ele. No total foram definidos 14 pontos de parada. Esses pontos são parâmetros de entrada para a roteirização dos veículos. Os clusters resultantes da primeira etapa, bem como os pontos de parada estão listados no Apêndice 1.

\subsection{ROTEIRIZAÇÃO}

Para resolver o problema de roteirização foi utilizado o programa IBM ILOG CPLEX, onde as funções mostradas na seção 2.1.2 foram programadas juntamente com os dados das quatro simulações propostas, comparando as soluções de cada uma e definindo a melhor. O cálculo da distância percorrida por cada rota e o tempo gasto para percorrer essa distância foram obtidos através do Google Maps. Os dados de entrada deste problema foram:

- Matriz de distâncias entre os pontos de parada, e entre a empresa e os pontos de parada (Apêndice 1)

- A quantidade de veículos;

- A capacidade de cada veículo.

Na primeira simulação foi considerado o uso de 4 vans com capacidade de 15 lugares cada. Os resultados obtidos nessa primeira simulação podem ser vistos na Tabela 3.4.1:

Tabela 3.4.1 - Rotas otimizadas na primeira simulação

\begin{tabular}{|c|c|c|c|c|}
\hline Veículos & Rota & $\begin{array}{c}\text { Capacidade } \\
\text { utilizada }\end{array}$ & $\begin{array}{c}\text { Distância } \\
\text { percorrida }\end{array}$ & Tempo \\
\hline 1 & $0-4-2-14-13-0$ & 15 & $107,35 \mathrm{~km}$ & $1 \mathrm{~h} 19 \mathrm{~min}$ \\
\hline 2 & $0-8-7-9-12-10-0$ & 14 & $117,9 \mathrm{~km}$ & $1 \mathrm{~h} 38 \mathrm{~min}$ \\
\hline 3 & $0-3-6-5-11-0$ & 15 & $105 \mathrm{~km}$ & $1 \mathrm{~h} 28 \mathrm{~min}$ \\
\hline 4 & $0-1-0$ & 6 & $81,9 \mathrm{~km}$ & $49 \mathrm{~min}$ \\
\hline \multicolumn{5}{c}{ Distância total percorrida } \\
\hline \multicolumn{5}{c}{ Fonte: O autor (2019) }
\end{tabular}

Na segunda simulação foram utilizados dois micro-ônibus com 26 lugares cada. Os resultados obtidos podem ser vistos na Tabela 3.4.2:

Tabela 3.4.2 - Rotas otimizadas na segunda simulação

\begin{tabular}{|c|c|c|c|c|}
\hline Veículos & Rota & $\begin{array}{c}\text { Capacidade } \\
\text { utilizada }\end{array}$ & $\begin{array}{l}\text { Distância } \\
\text { percorrida }\end{array}$ & Tempo \\
\hline 1 & $0-4-2-14-13-6-3-10-0$ & 25 & $113.25 \mathrm{~km}$ & $1 \mathrm{~h} 54 \mathrm{~min}$ \\
\hline 2 & $0-8-7-9-12-5-11-1-0$ & 25 & $120.1 \mathrm{~km}$ & $1 \mathrm{~h} 44 \mathrm{~min}$ \\
\hline \multicolumn{3}{|c|}{ Distância total percorrida } & \multicolumn{2}{|c|}{$233,35 \mathrm{~km}$} \\
\hline
\end{tabular}

Fonte: $O$ autor (2019)

$\mathrm{Na}$ terceira simulação foram utilizadas duas vans com capacidade de 15 lugares cada, e um micro-ônibus. Os resultados obtidos podem ser vistos na Tabela 3.4.3:

Tabela 3.4.3 - Rotas otimizadas na terceira simulação 


\begin{tabular}{|c|c|c|c|c|}
\hline Veículos & Rota & $\begin{array}{c}\text { Capacidade } \\
\text { utilizada }\end{array}$ & $\begin{array}{c}\text { Distância } \\
\text { percorrida }\end{array}$ & Tempo \\
\hline 1 (van) & $0-1-11-0$ & 9 & $89.4 \mathrm{~km}$ & $1 \mathrm{~h} 8 \mathrm{~min}$ \\
\hline 2 (van) & $0-4-2-14-13-0$ & 15 & $107,35 \mathrm{~km}$ & $1 \mathrm{~h} 19 \mathrm{~min}$ \\
\hline 3 (micro-ônibus) & $0-3-6-12-7-8-9-5-10-0$ & 26 & $120.9 \mathrm{~km}$ & $2 \mathrm{~h} 8 \mathrm{~min}$ \\
\hline Distância total percorrida & $317.65 \mathrm{~km}$ \\
\hline
\end{tabular}

$\mathrm{Na}$ última simulação foram utilizados um ônibus com 46 lugares e uma van com 15 lugares. Os resultados obtidos podem ser vistos na tabela 3.4.4:

Tabela 3.4.4 - Rotas otimizadas na quarta simulação

\begin{tabular}{|c|c|c|c|c|}
\hline Veículos & Rota & $\begin{array}{c}\text { Capacidade } \\
\text { utilizada }\end{array}$ & $\begin{array}{c}\text { Distância } \\
\text { percorrida }\end{array}$ & Tempo \\
\hline 1 (van) & $0-1-0$ & 6 & $81.9 \mathrm{~km}$ & $49 \mathrm{~min}$ \\
\hline 2 (ônibus) & $\begin{array}{c}0-3-4-2-14-13-7-8- \\
9-12-6-5-11-10-0\end{array}$ & 44 & $133.15 \mathrm{~km}$ & $2 \mathrm{~h} 38 \mathrm{~min}$ \\
\hline \multicolumn{3}{|c|}{ Distância total percorrida } & \multicolumn{2}{c|}{$215.05 \mathrm{~km}$} \\
\hline
\end{tabular}

Fonte: $O$ autor (2019)

A menor distância total acontece na situação com uma van e dois ônibus, porém a rota do ônibus dura duas horas e 38 minutos, o que é um tempo bastante alto para um funcionário gastar com o deslocamento para o trabalho. A opção com dois micro-ônibus aparece com a segunda menor distância total, e o tempo delas não ultrapassam as duas horas. Considerando que o valor de alugar um micro-ônibus é aproximadamente $16 \%$ maior do que o valor de alugar uma van, e o valor de alugar um ônibus é $33 \%$ maior do que alugar uma van, a opção com dois micro-ônibus e a opção com um ônibus e uma van são as menos custosas. Novamente, ressaltamos que a utilização de um ônibus e uma van possui uma rota com mais de duas horas. Levando ambos aspectos em consideração, é esperado que a rota mais vantajosa, considerando a distância total percorrida, o tempo de percurso das rotas e os custos, é a com dois micro-ônibus.

Para fins de comparação, o problema de roteirização também foi resolvido pelo método da "varredura" considerando uma frota composta por dois micro-ônibus, já que o mesmo mostrou-se a melhor simulação obtida pelo método exato. O método do vizinho mais próximo foi o método escolhido para minimizar as distâncias dentro de cada roteiro definido pelo método da "varredura. Os resultados obtidos do método da "varredura" seguido do vizinho mais próximo podem ser vistos na Tabela 3.4.5:

Tabela 3.4.5 - Rotas obtidas pelo método da "varredura"

\begin{tabular}{|c|c|c|c|c|}
\hline Veículos & Rota & $\begin{array}{c}\text { Capacidade } \\
\text { utilizada }\end{array}$ & $\begin{array}{c}\text { Distância } \\
\text { percorrida }\end{array}$ & Tempo \\
\hline 1 & $0-1-11-5-12-9-7-8-9-0$ & 25 & $124.4 \mathrm{~km}$ & $2 \mathrm{~h} 28 \mathrm{~min}$ \\
\hline 2 & $0-10-4-3-6-2-14-13-0$ & 25 & $115.95 \mathrm{~km}$ & $1 \mathrm{~h} 58 \mathrm{~min}$ \\
\hline \multicolumn{3}{|c|}{ Distância total percorrida } & \multicolumn{2}{c|}{$243.96 \mathrm{~km}$} \\
\hline
\end{tabular}

Fonte: $O$ autor (2019) 
Comparando a distância total percorrida obtida nos dois métodos, é possível notar que a distância obtida pelo método da "varredura" é maior do que o método sugerido no trabalho. Além disso, a duração total de uma das rotas no método da "varredura" ultrapassa duas horas, o que mostra que menor precisão de tal método.

\section{CONCLUSÃO}

\subsection{CONSIDERAÇÕES FINAIS}

Em geral, o problema de roteirização é complexo e, portanto, as soluções desenvolvidas geralmente são de difícil aplicação, necessitando de programas computacionais para resolvê-lo. O presente trabalho buscou criar um plano para roteirização de pessoas de forma simples para minimizar o custo gasto do transporte do funcionário até a sede de uma empresa, contabilizando a menor distância total possível levando a uma diminuição de custos e tempo de deslocamento para a empresa.

O método desenvolvido neste trabalho foi dividido em três etapas: a análise de cluster, a criação dos pontos de parada, com alocação dos funcionários, e a roteirização propriamente dita, realizada através do IBM ILOG CPLEX. Considerou-se 4 situações com diferentes veículos, comparando-as para definição da melhor rota. Constatou-se que a contratação de dois micro-ônibus com 26 lugares cada é a melhor opção em relação aos custos, distância total percorrida e tempo. Ainda, a título de comparação, o mesmo problema de roteirização foi resolvido pelo método da "varredura" e, a cada rota obtida, foi aplicada a heurística do vizinho mais próximo.

\section{REFERÊNCIAS BIBLIOGRÁFICAS}

[1] BRASIL. Lei $n^{\circ} 7.418$, de 16 de dezembro de 1985. Institui o Vale Transporte e dá outras providências.

[2] HAIR, J.F; BLACK, W.C.; BABIN, B.J.; ANDERSON, R.E.; TATHAM, R.L. Análise multivariada de dados. 6.ed. São Paulo: Bookman, 2006.

[3] FARAHANI, R. Z.; REZAPOUR, S.; DREZNER, T.; FALLAH, S. Competitive supply chain network design: An overview of classifications, models, solution techniques and applications. In: Omega international journal of management science. v. 45, pp. 92-118, jun, 2014.

[4] SLACK, N; CHAMBERS, S; JOHNSTON, R. Administração da Produção. 3.ed. São Paulo: Atlas, 2009.

[5] BALLOU, R. H. Gerenciamento da cadeia de suprimentos: logística empresarial. São Paulo: Bookman, 2006.

[6] ARENALES, M.; ARMENTANO, V.; MORABITO, R; YANASSE, H. Pesquisa Operacional. Rio de Janeiro: Elsevir, 2007.

[7] MILLER, C. E.; TUCKER, A. W.; ZEMLIN, R. A. Integer Programming Formulation of Traveling Salesman Problems. In: Journal of ACM. v. 7, pp. 326-329, oct. 1960.

[8] NOVAES, A.G. Logística e gerenciamento da cadeia de de distribuição. Rio de Janeiro: Elsevier, 2015.

[9] CHANDRASEKAR, C.; SRISANKAR, M. Clustering Large Databases Using Gmm. In: Int. Journal of Engineering Research and Applications. v. 4. pp. 209-211, april, 2014. 
[10] LINDEN, R. Técnicas de agrupamento. Revista de Sistemas de Informação da FSMA. Macaé, n. 4, pp. 18-36, 2009.

[11] SCHITTEKAT, P.; KINABLE, J.; SORENSEN, K.; SEVAUX, M.; SPIEKSMA, F.; SPRINGAEL, J. A metaheuristic for the school bus routing problem with bus stop selection. In: European journal of operational research. v. 229, p. 518-528, feb, 2013.

[12] FEO, T.A.; RESENDE, M.G.C. Greedy Randomized Adaptive Search Procedures. In: Journal of Global Optimization. v. 6, pp. 109-133, mar, 1995.

[13] PICHPIBUL, T.; KAWTUMMACHAI, R. An improved Clarke and Wright savings algorithm for the capacitated vehicle routing problem. In: ScienceAsia. v. 38, p.307-318, 2012.

[14] HERTZ, A; MITTAZ, M. A variable Neighborhood Descent Algorithm for the Undirected Capacitated Arc Routin Problem. In: Transportation Science. v. 35, nov, 2001.

[15] ZILLI, P.K. Análise de algoritmos heurísticos para problemas "ricos" de roteamento de veículos. 2011. 127 f. Dissertação (Mestrado em Ciência da Computação) - Instituto de Computação, UNICAMP, Campinas.

[16] BAGI, L.B. Algoritmo transgenético na solução do problema do caixeiro comprador. 2007. 169 f. Dissertação (Mestrado em Sistema e Computação) - Universidade Federal do Rio Grande do Norte, Natal.

[17] ESTEVAM, J. B. Heurística para o problema de roteamento de veículos capacitados - PRVC visando aplicação no Gerenciamento da Cadeia de Suprimentos. 2003. 117 f. Monografia (Bacharelado em Ciência da Computação) - Universidade Federal de Lavras, Lavras.

[18] SOUZA, L.V; SIQUEIRA, P.H. Técnicas da Pesquisa Operacional Aplicadas ao Problema do Transporte Escolar. In: SIMPÓSIO BRASILEIRO DE PESQUISA OPERACIONAL, XLV., 2013, Natal.

APÊNDICE I - PONTOS DE PARADA

\begin{tabular}{|c|c|c|c|c|}
\hline Clusters & $\begin{array}{l}\text { Endereço dos } \\
\text { funcionários }\end{array}$ & $\begin{array}{l}\text { Pontos de } \\
\text { parada }\end{array}$ & Endereço dos pontos de parada & $\begin{array}{c}\text { Auxílio } \\
\text { transporte }\end{array}$ \\
\hline \multirow{5}{*}{1} & $\begin{array}{l}\text { Rua jonatas de } \\
\text { vasconcelos, } 443\end{array}$ & Parada 1 & Rua Prof. João Medeiros, 901 & Não \\
\hline & $\begin{array}{l}\text { Rua Jonatas de } \\
\text { vasconcelos, } 294\end{array}$ & Parada 1 & Rua Prof. João Medeiros, 901 & Não \\
\hline & Rua Antônio Falcão & Parada 1 & Rua Prof. João Medeiros, 901 & Sim \\
\hline & Rua dr. raul Lafayette & Parada 1 & Rua Prof. João Medeiros, 901 & Não \\
\hline & Rua Jorge Couceiro & Parada 1 & Rua Prof. João Medeiros, 901 & Não \\
\hline \multirow{5}{*}{2} & Rua Ferreia Lopes & Parada 2 & Estr. do Arraial, 3140 & Não \\
\hline & Rua Estrela & Parada 2 & Estr. do Arraial, 3140 & Não \\
\hline & $\begin{array}{l}\text { Rua Astronauta Neil } \\
\text { Armostrong }\end{array}$ & Parada 2 & Estr. do Arraial, 3140 & Não \\
\hline & Av. Flor de Santana & Parada 2 & Estr. do Arraial, 3140 & Não \\
\hline & Rua Arnoldo Magalhães & Parada 2 & Estr. do Arraial, 3140 & Não \\
\hline
\end{tabular}




\begin{tabular}{|c|c|c|c|c|}
\hline \multirow{5}{*}{3} & Rua 10 de Novembro & Parada 3 & Av. Caxangá, 1252 & Não \\
\hline & Rua Estácio de Sá & Parada 3 & Av. Caxangá, 1252 & Não \\
\hline & Rua Ana Neri & Parada 4 & Rua Conde do Irajá, 408 & Não \\
\hline & Rua Marcos André & Parada 4 & Rua Conde do Irajá, 408 & Não \\
\hline & Rua Guilherme Pinto & Parada 3 & Av. Caxangá, 1252 & Sim \\
\hline \multirow{5}{*}{4} & Rua Venezuela & Parada 5 & $\begin{array}{l}\text { Av. Gov. Agamenon Magalhães, } \\
2656\end{array}$ & Não \\
\hline & Rua Santo Elias & Parada 5 & $\begin{array}{l}\text { Av. Gov. Agamenon Magalhães, } \\
2656\end{array}$ & Não \\
\hline & Rua Barão de Itamaracá & Parada 5 & $\begin{array}{l}\text { Av. Gov. Agamenon Magalhães, } \\
2656\end{array}$ & Não \\
\hline & Rua Amapá & Parada 6 & Rua quarenta e oito, 934 & Não \\
\hline & Rua Teles Jr. & Parada 6 & Rua quarenta e oito, 934 & Não \\
\hline \multirow{5}{*}{5} & Rua Canela & Parada 7 & $\begin{array}{l}\text { Avenida Senador Nilo Coelho, } \\
4463\end{array}$ & Não \\
\hline & Av. Senador Nilo Coelho & Parada 7 & $\begin{array}{l}\text { Avenida Senador Nilo Coelho, } \\
4463\end{array}$ & Não \\
\hline & Rua Peixe Agulha & Parada 8 & Av.Pan Nordestina, 3636 & Não \\
\hline & Rua Bolivar & Parada 12 & Avenida Beberibe, 838 & Não \\
\hline & Rua Sultão & Parada 8 & Av.Pan Nordestina, 3636 & Sim \\
\hline \multirow{5}{*}{6} & $\begin{array}{l}\text { Estrada do Forte do } \\
\text { Arraial Novo Bom Jesus }\end{array}$ & Parada 3 & Av. Caxangá, 1252 & $\operatorname{Sim}$ \\
\hline & Rua Brasileia & Parada 3 & Av. Caxangá, 1252 & Sim \\
\hline & Av. Tapajós & Parada 10 & $\begin{array}{l}\text { Av. Mal. Mascarenhas de Morais, } \\
2454\end{array}$ & Sim \\
\hline & Rua Alto da Bela Vista & Parada 3 & Av. Caxangá, 1252 & Sim \\
\hline & $\begin{array}{l}\text { Rua Visconde de } \\
\text { Jequitinhonha }\end{array}$ & Parada 1 & Rua Prof. João Medeiros, 901 & Sim \\
\hline \multirow{5}{*}{7} & Rua do Atlântico & Parada 11 & $\begin{array}{l}\text { Avenida Engenheiro Domingos } \\
\text { Ferreira, } 72\end{array}$ & Não \\
\hline & Rua Raul Azedo & Parada 11 & $\begin{array}{l}\text { Avenida Engenheiro Domingos } \\
\text { Ferreira, } 72\end{array}$ & Não \\
\hline & Rua Doze de Julho & Parada 11 & $\begin{array}{l}\text { Avenida Engenheiro Domingos } \\
\text { Ferreira, } 72\end{array}$ & Não \\
\hline & Rua Floriano Peixoto & Parada 5 & $\begin{array}{l}\text { Av. Gov. Agamenon Magalhães, } \\
2656\end{array}$ & $\operatorname{Sim}$ \\
\hline & Rua Itália & Parada 10 & $\begin{array}{l}\text { Av. Mal. Mascarenhas de Morais, } \\
2454\end{array}$ & Não \\
\hline \multirow[b]{3}{*}{8} & Av. Latão & Parada 12 & Avenida Beberibe, 838 & Sim \\
\hline & Rua dos Coqueiros & Parada 12 & Avenida Beberibe, 838 & Sim \\
\hline & Rua Nova Descoberta & Parada 13 & $\begin{array}{l}\text { Av. Norte Miguel Arraes de } \\
\text { Alencar, } 202\end{array}$ & $\operatorname{Sim}$ \\
\hline
\end{tabular}




\begin{tabular}{|c|c|c|c|c|}
\hline & $\begin{array}{l}\text { Av. José Américo de } \\
\text { Almeida }\end{array}$ & Parada 13 & $\begin{array}{l}\text { Av. Norte Miguel Arraes de } \\
\text { Alencar, } 202\end{array}$ & Não \\
\hline & Rua da Liberdade & Parada 13 & $\begin{array}{l}\text { Av. Norte Miguel Arraes de } \\
\text { Alencar, } 202\end{array}$ & $\operatorname{Sim}$ \\
\hline \multirow{5}{*}{9} & Rua Larga do Feitosa & Parada 9 & Estrada de Belém, 901 & Não \\
\hline & Rua São Caetano & Parada 9 & Estrada de Belém, 901 & Não \\
\hline & Rua Salvador de Sá & Parada 12 & Avenida Beberibe, 838 & Não \\
\hline & Rua Fortaleza & Parada 12 & Avenida Beberibe, 838 & Não \\
\hline & Rua Antônio Rangel & Parada 9 & Estrada de Belém, 901 & Não \\
\hline \multirow{5}{*}{10} & Rua Alto da União & Parada 13 & $\begin{array}{l}\text { Av. Norte Miguel Arraes de } \\
\text { Alencar, } 202\end{array}$ & Sim \\
\hline & Rua Anibal Cardoso & Parada 13 & $\begin{array}{l}\text { Av. Norte Miguel Arraes de } \\
\text { Alencar, } 202\end{array}$ & Sim \\
\hline & Estrada do Arraial & Parada 14 & Estrada do Arraial, 3666 & Não \\
\hline & Rua Evaristo da Veiga & Parada 14 & Estrada do Arraial, 3666 & Não \\
\hline & Estrada do Encanamento & Parada 14 & Estrada do Arraial, 3666 & Não \\
\hline
\end{tabular}

\section{APÊNDICE II - MATRIZ DE DISTÂNCIAS (EM KM) ENTRE OS PONTOS DE PARADA}

\begin{tabular}{|l|l|l|l|l|l|l|l|l|l|l|l|l|l|l|l|}
\hline & 1 & 2 & 3 & 4 & 5 & 6 & 7 & 8 & 9 & 10 & 11 & 12 & 13 & 14 & 15 \\
\hline 1 & 100 & 40.8 & 56.2 & 48.5 & 47.9 & 51.7 & 58 & 57.8 & 58 & 52.4 & 43.2 & 44.6 & 57.8 & 55 & 55.8 \\
\hline 2 & 41.1 & 100 & 13.3 & 11.2 & 11.3 & 12.3 & 12.1 & 19.4 & 19.6 & 13.3 & 3.6 & 4.2 & 13.3 & 18.9 & 13.8 \\
\hline 3 & 55.3 & 13.5 & 100 & 4.8 & 3.7 & 4.4 & 3.2 & 7.3 & 11.1 & 4.1 & 10.3 & 9.7 & 2.9 & 2.5 & 0.55 \\
\hline 4 & 49.9 & 11.6 & 5.2 & 100 & 2.4 & 4.8 & 4.7 & 10.4 & 14.1 & 5.7 & 7.9 & 7.9 & 6.1 & 9.4 & 5.8 \\
\hline 5 & 51.1 & 11.4 & 3.4 & 1.8 & 100 & 2.9 & 2.9 & 8.6 & 11.8 & 3.8 & 7.3 & 7.6 & 4.2 & 5.8 & 3.9 \\
\hline 6 & 48.9 & 10 & 3.6 & 3.5 & 3 & 100 & 2.4 & 8.3 & 10.5 & 3.2 & 8.1 & 6.2 & 3.1 & 6.1 & 4.1 \\
\hline 7 & 50.1 & 11.2 & 2.5 & 3.9 & 2.1 & 1.2 & 100 & 6.9 & 10.4 & 2.2 & 9.3 & 7.5 & 1.7 & 5.7 & 3 \\
\hline 8 & 59.8 & 20.9 & 6.9 & 14.4 & 8.6 & 11.4 & 6.4 & 100 & 2.7 & 5.2 & 19 & 17.1 & 5.2 & 8.5 & 7.4 \\
\hline 9 & 57.1 & 18.2 & 11.5 & 11.7 & 11.1 & 8.6 & 9.2 & 2.2 & 100 & 6.8 & 16.3 & 14.4 & 8.2 & 12.7 & 12 \\
\hline 10 & 50.3 & 12.8 & 4.2 & 6.3 & 3.2 & 2.4 & 1.7 & 5.6 & 8.5 & 100 & 10.9 & 9 & 1.3 & 5.8 & 4.8 \\
\hline 11 & 40.8 & 4.1 & 10.6 & 8.3 & 7.7 & 11.5 & 13 & 17.6 & 17.8 & 12.4 & 100 & 7.4 & 13.1 & 17.1 & 11.1 \\
\hline 12 & 44.4 & 3.8 & 10.3 & 9.4 & 8.9 & 9.3 & 9.6 & 16.8 & 17 & 10.3 & 5.3 & 100 & 10.3 & 12.7 & 10.8 \\
\hline 13 & 51.2 & 12.4 & 2.9 & 4.9 & 3.1 & 2.3 & 1.1 & 5.2 & 8.5 & 1.6 & 10.4 & 8.6 & 100 & 4.5 & 3.4 \\
\hline 14 & 53.6 & 17.7 & 5 & 9.1 & 7.9 & 8.1 & 6.9 & 11 & 18.9 & 7.7 & 17.1 & 18.7 & 6.6 & 100 & 4.7 \\
\hline 15 & 54.7 & 13.3 & 1.1 & 4.6 & 3.6 & 4.6 & 3.4 & 7.4 & 11.2 & 4.2 & 10.1 & 9.5 & 3.1 & 1.9 & 100 \\
\hline
\end{tabular}

